\title{
CMOS Sigma-Delta Thermal Modulator
}

\author{
W. R. M. Almeida, R. C. S. Freire \\ Federal University of Campina Grande \\ Campina Grande, PB, Brazil \\ willalmeida@hotmail.com, rcsfreire@dee.ufcg.edu.br
}

\author{
S. Y. C. Catunda \\ Federal University of Maranhao \\ Sao Luis, MA, Brazil \\ catunda@dee.ufma.br
}

\author{
H. Aboushady \\ Pierre et Marie Curie University \\ Paris, France \\ hassan.aboushady@lip6.fr
}

\begin{abstract}
This work presents the design of a $\mathbf{1}^{\text {st }}$-order sigmadelta thermal modulator using a thermoresistive sensor in its feedback loop, as the summing and integrating elements. The proposed system architecture allows the measurement of physical quantities that interact with the sensor, as temperature, thermal radiation and fluid velocity. Procedures for obtaining the design specifications needed for its modeling in VHDL-AMS are presented. A layout for the sigma-delta thermal modulator was developed for the integrated circuit technology CMOS $0.35 \mu \mathrm{m}$. Simulation results from this layout for temperature measurement are presented, showing that a resolution of 8.7 bits was attained, for a input signal frequency range of $12.5 \mathrm{kHz}$ and a sampling frequency of $25.6 \mathrm{MHz}$.
\end{abstract}

Keywords - sigma delta modulator, thermal, thermoresistive sensor, analog-to-digital converter, integrated circuits

\section{INTRODUCTION}

Thermoresistive sensors can be used for the measurement of thermal radiation, fluid velocity and temperature, using the principle of heat exchange with its surrounding environment. A mono-bit sigma-delta analog-to-digital converter (ADC) is composed by a difference amplifier, an integrator, a comparator and a digital-to-analog converter (DAC). In this work, the development of an integrated circuit that implements a thermal sigma-delta modulator, i.e., whose operating principle is based on heat exchange, is presented. Simulation results and analyses for the case of temperature measurement are also presented.

For the analysis of the modulator, some concepts related to the thermoresistive sensors and to the electrical equivalence principle are presented as follows.

\section{A. Thermoresistive sensors}

Even though all resistors vary their resistance with the temperature, only those having a considerable sensitivity with respect to the temperature, in a given operating range, are considered to be thermo-resistors. Thermoresistive sensors can be of the PTC (Positive Temperature Coefficient) type, generally metallic, or NTC (Negative Temperature Coefficient) type. The PTC sensors have an approximately linear temperature-resistance relationship, but low sensitivity. The NTC, conversely, have high sensitivity but a nonlinear temperature-resistance relationship.

The equation relating a PTC sensor electrical resistance with its temperature is approximately given by:

$$
R_{s}=R_{0}\left[1+\beta\left(T_{S}-T_{0}\right)\right]
$$

Where $R_{s}$ is the sensor resistance at the temperature $T_{s}, R_{0}$ is the sensor resistance at $0{ }^{\circ} \mathrm{C}$ and $\beta$ is the sensor temperature coefficient.

A thermoresistive sensor excited by an electrical current, exposed to the flow of a fluid, to thermal radiation and to the ambient temperature has a thermal equilibrium equation given by:

$$
R_{s} I_{s}^{2}+\alpha S H=h S\left(T_{s}-T_{a}\right)+m c \frac{d T_{s}}{d t} .
$$

Where $R_{S} I_{S}^{2}$ represents the electrical power dissipated by the sensor, $\alpha S H$ the thermal radiation absorbed by the sensor, $h S\left(T_{s}-T_{a}\right)$ the sensor energy lost to the ambient and $m c d T_{s} / d t$ the accumulated energy variation in the sensor, per time unit. In this equation, $S$ is the sensor surface area exposed to the radiation or to the flow of a fluid, $I_{S}$, is the electric current flowing through the sensor, $\alpha$ is the sensor coefficient of transmissivity-absorptivity, $h$ is the coefficient of heat transfer on the sensor surface, $m$ is its mass and $c$ is its specific heat; $T_{s}$ and $T_{a}$ are the sensor and ambient temperatures, respectively, and $t$ is the time.

For fluid with constant density and viscosity, the value of $h$ is given by the King's modified equation, by [1]:

$$
h=a+b \vartheta^{n} .
$$


Where $\vartheta$ is the fluid velocity and $a, b$ and $n$ are experimentally determined constants.

\section{B. Electric Equivalence Principle}

A feedback control system can be used for keeping the sensor heated at a constant temperature $T_{s}$, greater than the ambient temperature $T_{a}$. For the controlled system, a variation of one of the input quantities (ambient temperature, thermal radiation or fluid velocity) corresponds to a variation, of equal value and opposite signal, of the electric power dissipated by the sensor. Hence, it is possible to estimate the value of the variation of the input quantity from equations (1), (2) and (3), by measuring the value of the electric power variation [2-7]. This method is called electric equivalence principle.

With the purpose of obtaining more general expressions, the parameters from equations (1), (2) and (3), which describe the thermoresistive sensor static and dynamic behavior, where written as function of the time $t$, and for reducing the number of parameters, three substitutions where made: $I_{s}^{2}(t)=Y_{s}(t)$, $\alpha S=G_{H} \quad$ (thermal capacitance) and $S_{h}(t)=G_{t h}(t)$ (thermal conductance).

Considering the sensor temperature $T_{s}$ to be constant, once the employed method ensure this condition, equations (4), (5) and (6), each one giving evidence to a particular mesurand $\left(T_{a}(t), H(t), \vartheta(t)\right)$, can be obtained from the equations (1), (2) and (3), respectively, as:

$$
\begin{gathered}
T_{a}(t)=T_{s}-\frac{G_{H} H(t)+R_{s} Y_{s}(t)}{G_{t h}(t)}, \\
H(t)=\frac{R_{s} Y_{s}(t)+G_{t h}(t)\left[T_{s}-T_{a}(t)\right]}{G_{H}}, \\
\vartheta(t)=\left\{\frac{1}{b}\left[\frac{G_{H} H(t)+R_{s} Y_{s}(t)}{T_{s}-T_{a}}-a\right]\right\}^{\frac{1}{n}} .
\end{gathered}
$$

\section{Electric Equivalence Principle}

A mono-bit $\Sigma \Delta$ modulator is composed by an integrator, a quantizer and a feedback loop with a DAC, as represented in Figure 1. The system is based on the principle of oversampling, where the input signal sampling frequency is $N$ times greater

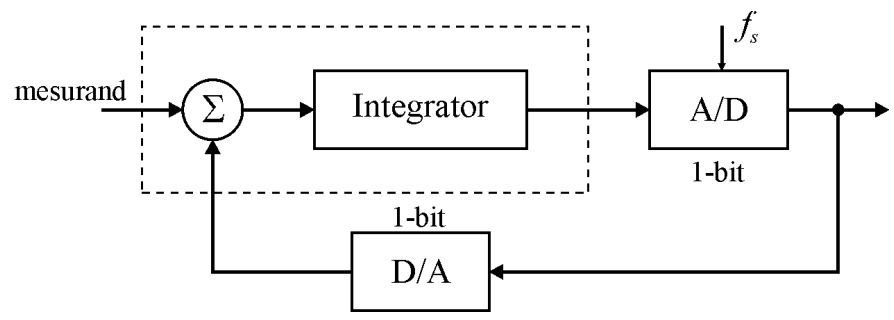

Figure 1. Block diagram of a $1^{\text {st }}$-order $\Sigma \Delta$ modulator than the Nyquist frequency.

The functions of sum and integration can be performed by a thermoresistive sensor included in the $\Sigma \Delta$ modulator loop. Hence, it is possible to carry out the conditioning of the sensor output signal and its analog-to-digital conversion simultaneously. This circuit topology may be called sigmadelta thermal modulator, since all the processing is carried out considering the sensor thermal interaction with the quantity to be measured, with the sensor excitation made by Joule effect feedback.

\section{II. $\Sigma \Delta$ THERMAL MODULATOR}

Figure 2 shows the schematic diagram of a sigma-delta thermal modulator, developed from the model presented in Figure 1. The input $X(t)$ corresponds to the mesurand, which can be $T_{a}(t), H(t)$ or $\vartheta(t)$. The DAC was substituted by a constant-period pulse generator where the pulse width can assume only two values, according to the value of the input bit. The summing and integrating blocks are implemented by the sensor $R_{s}$, which carries out these operations by thermal interaction between the electric power, $R_{s} I_{S}^{2}$, the absorbed thermal radiation, $\alpha S H$ and the sensor energy lost to the ambient by time unit $h S\left(T_{s}-T_{a}\right)$, which is consequence of the ambient temperature $T_{a}$ and of the forced convection, with $h=a+b \vartheta^{n}$. The 1-bit ADC is a comparator between the voltage across the sensor, when its current value is high (different from zero), and a reference voltage $V_{\text {ref }}$. This reference voltage imposes the sensor operating temperature.

In the following, design considerations of this sigma-delta thermal modulator are presented.

\section{A. Micro-sensor}

The choice of the sensor was based mainly on the proposal of integrating the sensor in the sigma-delta modulator loop (using CMOS technology) in the same IC and, also, because this type of sensor uses less power in its measurement system, when compared to metallic PTC sensors.

The parameters that characterize the sensor, essential for the estimation of fluid velocity, are: area $S=4 \mathrm{~nm}^{2}$, length $L=200 \mu \mathrm{m}$, width $W=20 \mu \mathrm{m}, \beta=0.000784{ }^{\circ} \mathrm{C}^{-1}, n=0.5$, $a=32325 \mathrm{~W} \cdot{ }^{\circ} \mathrm{C}^{-1} \cdot \mathrm{m}^{-2}, b=25575 \mathrm{~W} \cdot \mathrm{s}^{0.5} \cdot{ }^{\circ} \mathrm{C}^{-1} \cdot \mathrm{m}^{-2.5}, R_{0}=102 \Omega$ and $m c=292.56 \cdot 10^{-12} \mathrm{~J} \cdot{ }^{\circ} \mathrm{C}^{-1}[8,9]$. The sensor operating temperature was chosen to be $T_{s}=76{ }^{\circ} \mathrm{C}$, which results in $R_{s}=107.74 \Omega$, from equation (1). With these parameters and the equations modeling the operation of the micro-sensor, it

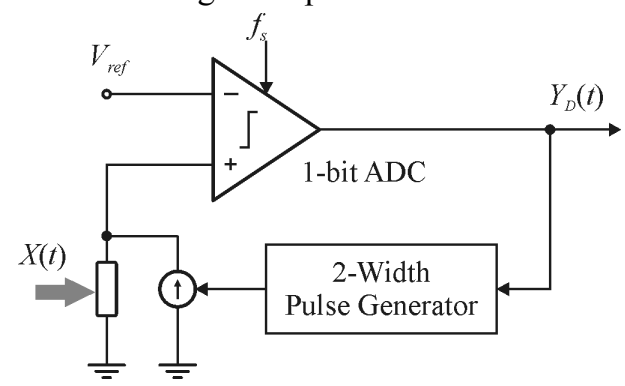

Figure 2. Block diagram of a $1^{\text {st }}$-order $\Sigma \Delta$ thermal modulator. 
was possible to carry out simulations and to validate the circuit of the modulator.

\section{SYSTEM MODELLING}

A model of a feedback measurement system with a thermoresistive sensor was developed in VHDL-AMS, based on the $\Sigma \Delta$ modulator presented in Figure 2. Only considerations for the temperature measurement are presented in the following.

\section{A. Temperature Measurement}

Considering the ambient temperature as the sensor only input and the sensor heated by the Joule effect, in static regime and thermal equilibrium condition, the following can be written:

$$
I_{s}^{2}=\frac{G_{t h}\left(T_{s}-T_{a}\right)}{R_{s}} .
$$

As $G_{t h}, T_{s}$ and $R_{s}$ are constant, (7) describes a straight line with two variables, $I_{s}^{2}$ and $T_{a}$. As the interactions of the sensor with the quantities are thermal, and the $I_{s}$ current is pulsed with a period much smaller than the sensor time constant, the rms value of $I_{s}$ is used for the assessment of the feedback system, which is denominated as $I_{r m s}$.

For (7), one can obtain the graphic of Figure 3.a for the squared current $I_{r m s}^{2}$ as function of the ambient temperature $T_{a}$. It can be observed that the maximum value of $I_{r m s}^{2}\left(I_{r m s \max }^{2}\right)$ happens for the minimum value of $T_{a}\left(T_{a \mathrm{~min}}\right)$ and that the minimum value of $I_{r m s}^{2}\left(I_{r m s \text { min }}^{2}\right)$ happens for the maximum value of $T_{a}\left(T_{a \text { max }}\right)$. The current pulses (Figure 3.b) have only

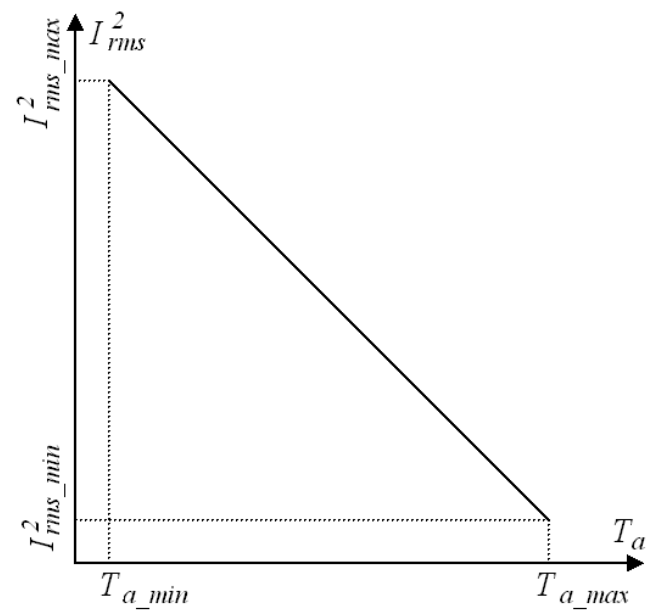

a)

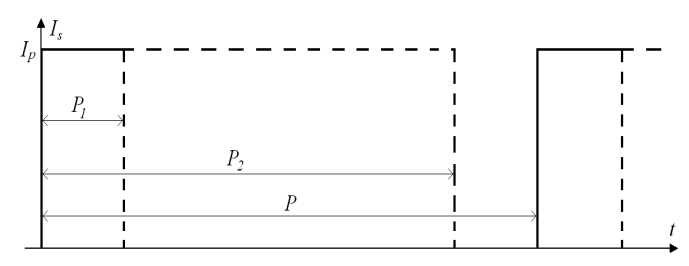

b) two widths $P_{1}$ and $P_{2}$ and a $P$ period. The pulses with $P_{1}$ width must correspond to an input of ambient temperature greater than or equal to $T_{a \text { max }}$ and the pulses with $P_{2}$ width must correspond to an input of ambient temperature less than or equal to $T_{a \_ \text {min }}$.

For current pulses with duration of $P_{y}$ ( $y$ equals to 1 or 2 ), period of $P$ and amplitude of $I_{p}$, the rms value of the squared current is

$$
I_{r m s}^{2}=I_{p}^{2} \frac{P_{y}}{P}
$$

The relationship between the maximum and minimum current pulse durations (ratio_P) is:

$$
\text { ratio } \_=\frac{P_{2}}{P_{1}}=\frac{I_{r m s_{-} \max }^{2}}{I_{r m s_{-} \min }^{2}} .
$$

Substituting (8) in (7) gives:

$$
I_{p}^{2} \frac{P_{y}}{P}=\frac{G_{t h}}{R_{s}}\left(T_{s}-T_{a}\right)
$$

Using $I_{r m s}^{2}$ relationship for the values of $T_{a_{-} \min }$ and $T_{a_{-} \max }$, i.e., $I^{2}{ }_{r m s_{-} \max } I^{2}{ }_{r m s_{-} \min }$, from (9) and (10), one has:

$$
\text { ratio_ } P=\frac{T_{s}-T_{a_{-} \min }}{T_{s}-T_{a_{-} \max }} .
$$

Rewriting (11) for $T_{s}$, gives:

$$
T_{s}=\frac{\text { ratio } \_P T_{a_{-} \max }-T_{a_{-} \min }}{\text { ratio__ } P-1} .
$$

Choosing an operating temperature for the sensor and knowing the equation parameters relating the resistance to the temperature, one can determine the value of $R_{s}$ from (1).

If the values of $R_{s}$ and of $T_{s}$ are known, it is possible, from (7), to determine the maximum and minimum values of the squared rms current:

$$
I_{r m s_{-} \max }^{2}=\frac{G_{t h}}{R_{s}}\left(T_{s}-T_{a_{-} \min }\right) .
$$

and

$$
I_{r m s_{-} \min }^{2}=\frac{G_{t h}}{R_{s}}\left(T_{s}-T_{a_{-} \max }\right) .
$$

Figure 3. a) $I_{r m s}{ }^{2}$ as function of $T_{a}$ for $T_{s}$ constant; b) Current pulses. 
The apparent time constant of the sensor, $\tau_{a}$, as function of the mean squared current is given by [9]:

$$
\tau_{a}=\frac{C_{t h}}{k_{t} I_{r m s_{-} a v}^{2}-G_{t h}} .
$$

Where $k_{t}$ is the derivative of the resistance as function of the temperature in the operating point.

For the determination of this apparent time constant of the sensor a mean operating temperature was chosen, corresponding to the value of the average squared current, given by:

$$
I_{r m s_{-} a v}^{2}=\frac{I_{r m s_{-} \max }^{2}+I_{r m s_{-} \min }^{2}}{2} .
$$

For the sensor to present the characteristics of an integrator, the modulator sampling period $(P)$ must be much less than the sensor apparent time constant. For assuring this integrator characteristic, it was adopted the relationship of $P=\tau_{d} / 10$ [9].

The value of $I_{p}$ (Figure 3.a) can be determined from one of the values of $I_{r m s}^{2}$, as for instance:

$$
I_{p}=\left(\frac{I_{r m s \_ \text {max }}^{2} P}{P_{2}}\right)^{0.5} .
$$

Knowing the value of $I_{p}$, the value of the reference voltage of the $\Sigma \Delta$ modulator can be determined by $V_{r e f}=I_{p} R_{s}$.

Once the sensor parameters are known, the measurement range values are defined, the PWM signal widths relationship are fixed and the initial operating conditions are determined $\left(V_{\text {ref }}\right.$ and $I_{p}$ ) for the $\Sigma \Delta$ thermal modulator operating as a thermometer, a routine was developed for determining the necessary parameters for the functional simulation in the

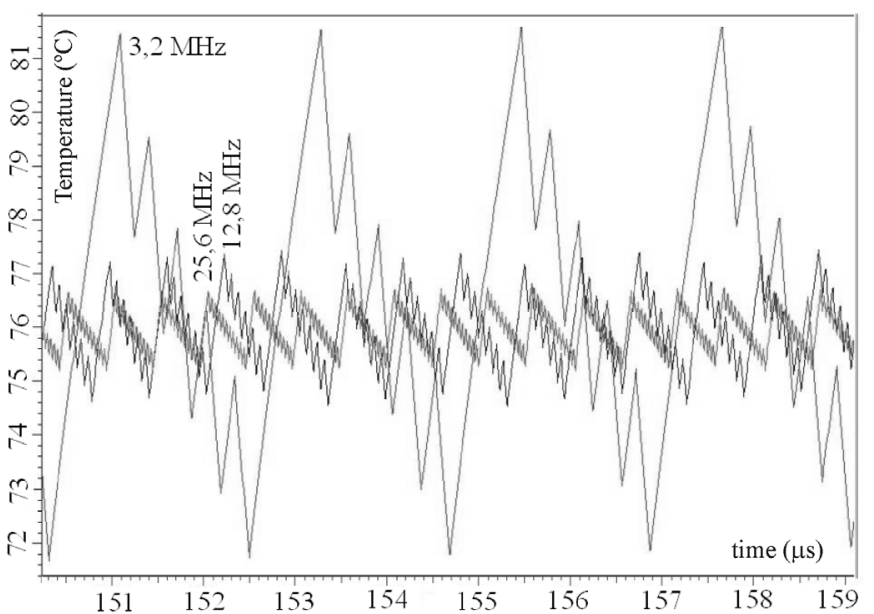

Figure 4. Sensor temperature variation.
VHDL-AMS environment.

\section{B. VHDL-AMS Simulation}

The sigma-delta thermal modulator was simulated in the VHDL-AMS environment from the implementation of its mathematical model. From the obtained simulation results, an analysis of the performance was carried out considering as parameters the number of bits and the signal-to-noise relationship of the system. These considerations are presented as follows.

As the main idea is to use the structure based on the $\Sigma \Delta$, where a micro-sensor of the thermoresistive type is part of the feedback loop, a high functional level model for this modulator was implemented in the VHDL-AMS environment.

Calculations of the Fast Fourier Transform (FFT) and of the signal to noise ratio (SNR) of the modulator output signal where carried out using simulated results obtained at the comparator output.

The ADMS simulator was employed for simulating the $\Sigma \Delta$ thermal modulator code, developed in the VHDL-AMS description language. Considering the signal frequency range to be $12.5 \mathrm{kHz}$, a sinusoidal temperature signal with a frequency of $1388 \mathrm{~Hz}$ was applied at the input of the modulator, for oversampling frequencies of $3.2 \mathrm{MHz}$, 12.8 MHz and 25.6 MHz (oversampling ratios of 128, 512 and 1024 , respectively). The obtained values of the SNR were $43.7 \mathrm{~dB}, 52.8 \mathrm{~dB}$ and $57.5 \mathrm{~dB}$ with respective resolutions of 7 bits, 8.5 bits and 9.3 bits.

The temperature of the sensor must be approximately constant, around $76{ }^{\circ} \mathrm{C}$. The graph shown in Figure 4 presents the temperature variation for the modulators with oversampling frequencies of $3.2 \mathrm{MHz}, 12.8 \mathrm{MHz}$ and $25.6 \mathrm{MHz}$. It can be verified that the sensor temperature variation is smaller for greater values of the oversampling frequency.

\section{LAYOUT DEVELOPMENT}

The sigma-delta thermal modulator functional model blocks where implemented with circuits in the transistor level. For the dimensioning of the circuit transistors it was used the OCEANE tool and its CONDIAC module, which are dedicated to the dimensioning of analog CMOS integrated circuits [10]. The employed CMOS technology was the TMSC $0.35 \mu \mathrm{m}$.

The sigma-delta thermal modulator circuit layout is presented in Figure 5, and has the dimensions of $337 \mu \mathrm{m} \mathrm{x}$ $265.5 \mu \mathrm{m}$. This layout was realized manually in order to allow a greater symmetry of its composing blocks and to optimize its performance.

Parameter extraction was performed for the circuit layout presented in Figure 5 and simulations where carried out for the circuit with these extracted parameters. The previously described procedures for the simulation and analysis of the $\Sigma \Delta$ modulator in the VHDL-AMS environment where repeated here. The oversampling frequency used was $25.6 \mathrm{MHz}$

In Figure 6, simulation results for the extracted circuit are presented for values of the SNR and SNDR (Signal-to-noise + 
distortion ratio) as function of the sinusoidal input signal with variable amplitude. With the SNR value equal to $54.6 \mathrm{~dB}$, the effective resolution of 8.7 bits can be obtained. The maximum value of the SNDR obtained for this simulation was $43.7 \mathrm{~dB}$.

Comparing the obtained simulation results in this simulation, referring to the performance analysis, with the simulation results obtained from the VHDL-AMS model, one can verify that the resolution values for the later are approximately $5 \%$ greater than for the former. This percent difference is acceptable.

The dissipated power value found for the simulation is around $29.3 \mathrm{~mW}$.

\section{CONCLUDING REMARKS}

The design of a $1^{\text {st }}$-order $\Sigma \Delta$ thermal modulator containing a PTC type thermoresistive sensor in its feedback loop, with functional characteristics corresponding to the adder/integrator, was presented in this work. The functional model of the sigmadelta thermal modulator was firstly developed and simulated in VHDL-AMS environment, in order to verify the design viability, performance and conformity with the model equations.

A layout was developed and simulations were carried out for the TSCM $0.35 \mu \mathrm{m}$ technology. Simulation results obtained from this circuit, designed in the transistor level and using extracted layout parameters, were compared and agreed with results obtained from the VHDL-AMS model, with respect to the SNR values and number of bits. For an oversampling ration of 1024 a resolution of 8.7 bits was obtained.

\section{REFERENCES}

[1] R. P. C. Ferreira, R. C. S. Freire, G. S. Deep, J. S. Rocha Neto, A. Oliveira, Hot-Wire Anemometer With Temperature Compensator Using Only One Sensor. IEEE Transactions on Instrumentation and Measurement, USA, V. 50, N. 4, P. 954-958, 2001.

[2] L. S. Palma, A. Oliveira, A.S. Costa, A.Q. Andrade Jr., C.V.R. Almeida, M.E.P.V. Zurita, R.C.S. Freire, Implementation of a feedback $I^{2}$ -

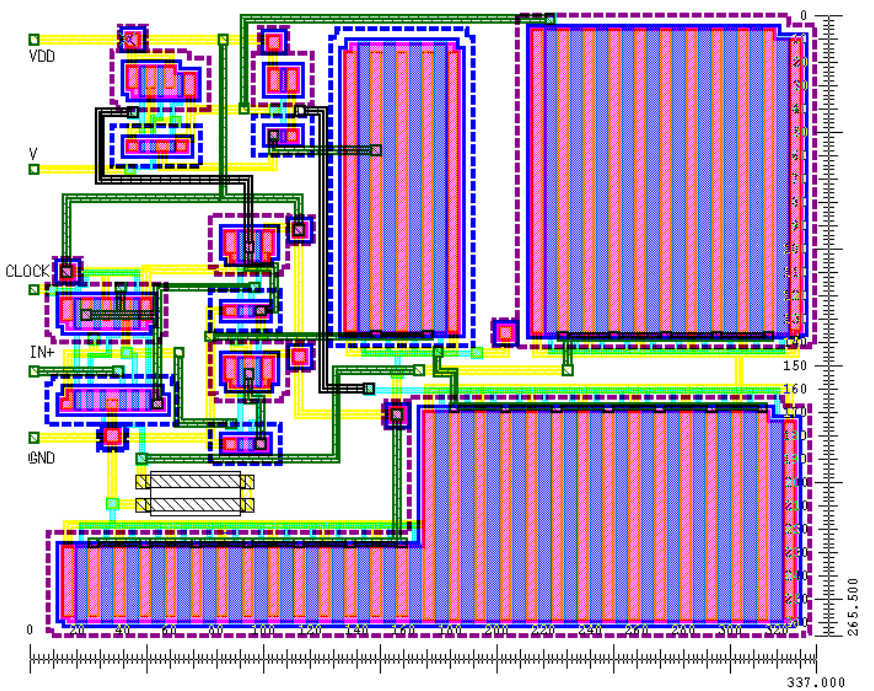

Figure 5. $\Sigma \Delta$ thermal modulator circuit layout. controlled constant temperature environment temperature meter, Sensors 3 (10) (2003) 498-503.

[3] A. Oliveira, G. S. Deep, A.M.N. Lima, R.C.S. Freire, A feedback $I^{2}$ controlled constant temperature solar radiation meter, in: Proc. IEEEIMTC, vol. 2, 1998, pp. 1062-1066.

[4] R.C.S. Freire, G.S. Deep, C.C. Farias, Electrical equivalence solar radiometer configurations, in: Proc. XI Congresso Brasileiro de Automatica, vol. 3, 1996, pp. 1249-1254.

[5] L.S. Palma, A. Oliveira, A. S. Costa, R.C.S. Freire, A.C. Lima, $A$ Constant Temperature Operation Thermoresistive Sigma-Delta Solar Radiometer. Proceedings 10th IMEKO TC7 International Symposium on Advances of Measurement Science. 2004. v. 1. p. 199-204.

[6] R.P.C. Ferreira, R.C.S. Freire, G.S. Deep, J.S. Rocha Neto, A. Oliveira, Fluid temperature compensation in a hot wire anemometer using a single sensor, in: Proc. IEEE-IMTC,vol. 1, 2000, pp. 512-517.

[7] H. Fujita, T. Ohhashi, M. Asakura, M. Yamada, K. Watanabe, A Thermistor Anemometer for Low-Flow-Rate Measurements, IEEE Trans. Instrum. Meas. 44 (3) (1995) 779-782.

[8] C.O. Júnior. Desenvolvimento de Micro-Aquecedores Compativeis com Tecnologia de Microeletrônica para Aplicação em Transdutores Térmicos. 2003. Dissertação (Electrical Engineering Mater's Dissertation) - Universidade Estadual de Campinas.

[9] W.R.M. Almeida, Modulador $\Sigma \Delta$ Térmico: Modelagem, Simulação e Concepção, PhD Thesis, 2009, Universidade Federal de Campina Grande, Brazil.

[10] J. Porte. COMDIAC: Compilateur de Dispositifs Actifs, Reference Manual. ENST- Paris Ecole Nationale Superieure des Télécommunications, France, 1997.

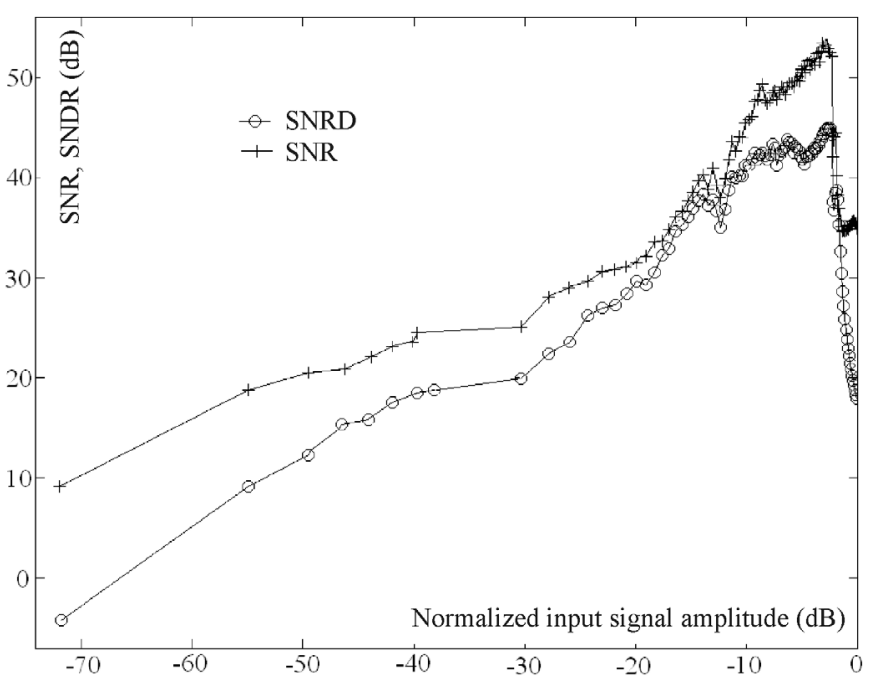

Figure 6. $\Sigma \Delta$ thermal modulator SNR and SNDR for the trasistor-level simulation. 